\title{
Salvia miltiorrhiza aqueous root extract plays an important role in improving locomotor activity in rats with spinal cord injury
}

\author{
Ji-jun Liu ${ }^{1}$, Xin-wen Wang ${ }^{1}$, Shu-fang $W^{2}{ }^{2}$, Qi-ning $W^{1}$ and Ding-jun Hao ${ }^{{ }^{*}}$ \\ ${ }^{1}$ Department of Spine Division, Xi'an Jiaotong University-affiliated Red Cross Hospital, ${ }^{2}$ Department of Translational Medicine, \\ Xi'an Jiaotong University, Xi'an 710054, China
}

*For correspondence: Email: haodingjun@hotmail.com; Tel/Fax: 0086-29-87800002

Received: 18 January 2016

Revised accepted: 6 July 2016

\begin{abstract}
Purpose: To investigate the activity of the aqueous root extract of Salvia miltiorrhiza (S. miltiorrhiza) (Lamiaceae), collected from Anhui Province, China, for the treatment of spinal cord injury (SCl) in Sprague-Dawley (SD) rats.

Methods: In total, 30 adult rats were selected and divided into three groups; normal control, untreated and treated. Aqueous root extract of S. miltiorrhiza was introduced intraperitoneally to the treated group. Basso, Beattie and Bresnahan rating scale (BBB) was used to evaluate improvement in locomotor activity after SCl. Total RNA was extracted from tissue sections using Sepasol (NacalaiTesque) and RNA samples were reverse-transcribed using M-MLV reverse transcriptase. BioSense SC-810 Gel Documentation System and Gel-Pro 3.1 software were employed for the analysis of band intensity. Results: A significant reduction in SCl cavity area was observed in the S. miltiorrhiza extract-treated group, relative to the untreated group, after 11 days $\left(0.10 \pm 0.05 \mathrm{~mm}^{2}\right.$ treated vs. $0.30 \pm 0.01 \mathrm{~mm}^{2}$ untreated). Treatment with root extract also improved the BBB scores; the treated group scored 15, compared to a score of 8 for the untreated group. In addition, the degradation of neurons at the site of injury in the spinal cord was reduced in the treated group compared to the untreated group. Treatment with S. miltiorrhiza aqueous root extract also significantly increased the expression of platelet-derived growth factor-B (PDGF-B) mRNA ( $p<0.01)$.

Conclusion: These data suggest that, in addition to other pharmacological activities, S. miltiorrhiza extract has therapeutic potential for the treatment of neuronal degeneration following SCI.
\end{abstract}

Keywords: Salvia Miltiorrhiza, Neurons, Spinal cord injury, Locomotor capacity, Platelet-derived growth factor- $B$, Basso, Beattie and Bresnahan rating scale

Tropical Journal of Pharmaceutical Research is indexed by Science Citation Index (SciSearch), Scopus, International Pharmaceutical Abstract, Chemical Abstracts, Embase, Index Copernicus, EBSCO, African Index Medicus, JournalSeek, Journal Citation Reports/Science Edition, Directory of Open Access Journals (DOAJ), African Journal Online, Bioline International, Open-J-Gate and Pharmacy Abstracts

\section{INTRODUCTION}

Spinal cord injury (SCl), which is primarily caused by traffic accidents and falls from high altitude, is a frequently encountered global health problem [1]. The pathology of SCl can be divided into two stages, primary and secondary injury, involving (1) compression of spinal cord tissues and (2) apoptosis of cells at the site of lesions, respectively [2,3]. The permanent loss of neurological functions and the associated lifethreatening complications make $\mathrm{SCl}$ a major clinical issue across the globe [4]. SCl involves loss of either partial or complete neuronal sensory and motor functions, because of the failure of neuronal regeneration. Axonal regeneration is inhibited by autonomous cellular factors $[5,6]$, astroglial scarring $[7,8]$ and by the 
accumulation of central nervous system (CNS) myelin $[8,9]$.

There are cells in the CNS that produce plateletderived growth factor (PDGF), which has been demonstrated to bind to NMDA receptors [10]. It is reported that PDGF plays a vital role in recovery following CNS injury [10]. The mechanism of action of PDGF involves activation of tyrosine kinase receptors, including PDGF- $\alpha R$ and PDGF- $\beta R$. PDGF has also been identified to promote the growth of axons and to maintain the integrity of neurons [11]. PDGF isoforms induce mitosis in glial cells, thereby conferring a protective effect on the brain.

Natural products, either in the form of extracts or isolated constituents, have been used for the treatment of various disorders. Extracts from plants like Lycium barbarum (Solanaceae) and $S$. miltiorrhiza root (Lamiaceae) have been shown to possess neuroprotective activities $[12,13]$. It has been reported that the alcoholic root and rhizome extract of $S$. miltiorrhiza (Lamiaceae) protects the eyes of elderly people and provides relief from strain and pain $[14,15]$. The present study was designed to investigate the effect of $S$. miltiorrhiza root extract on rats with $\mathrm{SCl}$. It was observed that S. miltiorrhiza (Lamiaceae) root extract improved locomotor activity, indicating that it may be a promising treatment option for $\mathrm{SCl}$.

\section{EXPERIMENTAL}

\section{Animals}

Thirty adult (8 weeks of age) Sprague-Dawley (SD) rats were obtained from Guangdong Medical Laboratory Animal Co. (Guangdong, China). The animals were acclimatized to the laboratory atmosphere for 1 week prior to commencement of the experiment, and were housed under $12 \mathrm{~h}$ dark/light cycle conditions with free access to water. Experimental procedures were carried out according to the guidelines approved by the Animal care and use committee of Xi'an Jiaotong University (approval no. 2014/10C-25). The studies were carried out in accordance with the Directive Office of Laboratory Animal Welfare, National Institutes of Health (OLAW/NIH 2002) on the guidelines for care and safe use of laboratory animals for scientific purposes [16].

\section{Plant extract}

Initially, the plant samples were collected from Gunagdong, Sichuan, and Shangdong Provinces and all specimens were examined, identified and authenticated by Dr. H.W. Wang, Plant Taxonomist, China Agricultural University. The specimens were registered and the voucher specimen (no. HKLPR/IBA/CAS/consult/-201403/438/161) deposited in the Herbarium of the Key Laboratory of Plant Resources, Institute of Botany, Chinese Academy of Sciences.

S. miltiorrhiza (Lamiaceae) plant root was collected, washed with distilled water, dried and ground to form a fine powder. The powder was added to a mixture of distilled water and methanol (1:1 ratio) and macerated for 2 days, after which the solvent mixture was evaporated and filtered through Whatman paper to obtain a pure extract.

\section{Reagents and chemicals}

Dimethyl sulphoxide (DMSO) and 3,3'diaminobenzidine hydrochloride (DAB) were purchased from Sigma Co. (St. Louis, MO, USA). Antibodies for PDGF-B and biotinylated goat anti-rabbit secondary antibody were obtained from Santa Cruz Biotechnology, Inc. (Santa Cruz, CA, USA).

\section{Treatment strategy}

The rats were divided randomly into three groups (normal control, untreated and treated) of 10 animals each. The animals in the untreated and treated groups were anesthetized by intra peritoneal injection of sodium pentobarbital (50 $\mathrm{mg} / \mathrm{kg}$ ), before exposure of the spinal cord. A contusion injury was carefully made in the spinal cord of rats in both groups using a weight-drop device [17]. The incision was closed by sterile suturing and the animals were kept in their respective marked cages under sterilized conditions.

Animals in the treated group were injected with $10 \mu \mathrm{M}$ doses of $S$. miltiorrhiza root extract every other day for 10 days. Animals in the untreated and normal control groups were injected with the same volume $(5 \mathrm{~mL} / \mathrm{kg})$ of normal saline. On day 11 , the animals were placed on the floor and their locomotor ability was monitored for $10 \mathrm{~min}$ [18]. Locomotor capacity was scored based on the Basso, Beattie and Bresnahan rating scale (BBB).

\section{Examination of pathological changes [10]}

On day 11 , four animals from each group were anesthetized and a surgical incision was performed to open the thoracic cavity. A segment of the thoracic spinal cord from T6 to T14 was removed from each animal, washed in normal 
saline and fixed in $4 \%$ paraformaldehyde in 0.1 $\mathrm{M}$ phosphate-buffered saline (PBS) for $5 \mathrm{~h}$. The tissue sections were rinsed twice in PBS and then incubated for 45 min with $1 \%$ bovine serum albumin (BSA). The sections were then incubated with primary antibodies (anti-PDGF-B primary antibody) in BSA for $12 \mathrm{~h}$. Following incubation, the sections were washed again with PBS and incubated with biotinylated goat antirabbit secondary antibody.

The sections were then treated with avidin-horse radish peroxidase (HRP) solution. Visualization was achieved using DAB and hydrogen peroxide dissolved in PBS. The paraffin-embedded sections were heated in xylene, and hydrated in a gradient of ethyl alcohol. Finally, the sections were mounted onto poly-L-lysine-coated slides using Permount ${ }^{\mathrm{TM}}$. The lesion sites were examined under a microscope using H\&E staining.

\section{Analysis of PDGF-B mRNA expression [18]}

Total RNA was extracted from tissue sections using Sepasol (NacalaiTesque) according to the manufacturer's protocol. Moloney-murine leukemia virus (M-MLV) reverse transcriptase (Life Technologies Japan Ltd., Tokyo, Japan) was used to reverse-transcribe the RNA samples, using a thermal cycler (Takara PCR Thermal Cycler S; Takara Bio Inc., Shiga, Japan). The mRNA expression levels were evaluated using a real-time quantitative RT-PCR assay and a Light Cycler System (Roche Diagnostics, Mannheim, Germany).

Separation of the PCR products was performed using electrophoresis on a $2.0 \%$ agarose gel. A BioSense SC-810 Gel Documentation System (Shanghai Bio-Tech Co., Ltd., Shanghai, China) and Gel-Pro software (ver. 3.1; Media Cybernetics, Inc., Bethesda, MD, USA) were employed for the analysis of the intensity of the bands.

\section{Statistical analysis}

All of the experiments were performed in triplicate and the data are presented as mean \pm standard deviation (SD). Statistical analysis of the data was performed using SPSS for Windows software (ver. 16.0; SPSS Inc., Chicago, IL, USA).

Statistically significant differences were identified using one-way analysis of variance (ANOVA). Differences were considered significant at $p<$ 0.05 .

\section{RESULTS}

\section{S. miltiorrhiza root extract increased the BBB score}

The animals belonging to the normal control, untreated and treated groups were examined for locomotor activity on day 11 post-surgery. It was observed that animals in the untreated and treated groups suffered from hind limb paralysis and were unable to walk normally in the initial days after the saline or root extract injection regimen was initiated, whereas animals in the normal control group walked normally. However, treatment with $S$. miltiorrhiza root extract improved recovery from hind limb paralysis; animals in the treated group started to walk normally after day 10 . The BBB score in the $S$. miltiorrhiza root extract-treated group improved, reaching 15 points, compared to the 8 points scored by the untreated group of rats (Fig 1). These findings indicate that $S$. miltiorrhiza root extract has a therapeutic effect on rats following SCl $(p<0.01)$.

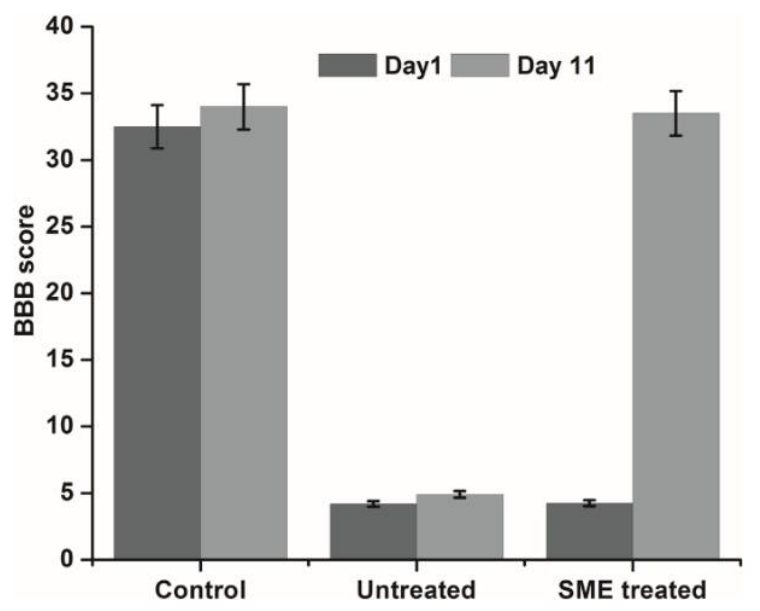

Figure 1: Effect of Salvia miltiorrhiza (S. miltiorrhiza) root extract on Basso, Beattie and Bresnahan (BBB) rating scale scores on days 1 and 11 after surgery. The animals in the control and untreated group were injected with normal saline, whereas those in the treated group were administered $10 \mu \mathrm{M}$ doses of $S$. miltiorrhiza root extract for 10 days

The results from H\&E staining revealed that the spinal cord tissues of the untreated group of animals featured vacuolar regions, reduced numbers of gliocytes and neuronal apoptosis. However, treatment of the rats with $S$. miltiorrhiza root extract prevented both the formation of vacuoles and neuronal apoptosis. The animals in the treatment group were found to possess large numbers of gliocytes in the spinal cord at the site of injury (Fig. 2).

Trop J Pharm Res, August 2016; 15(8): 1669 


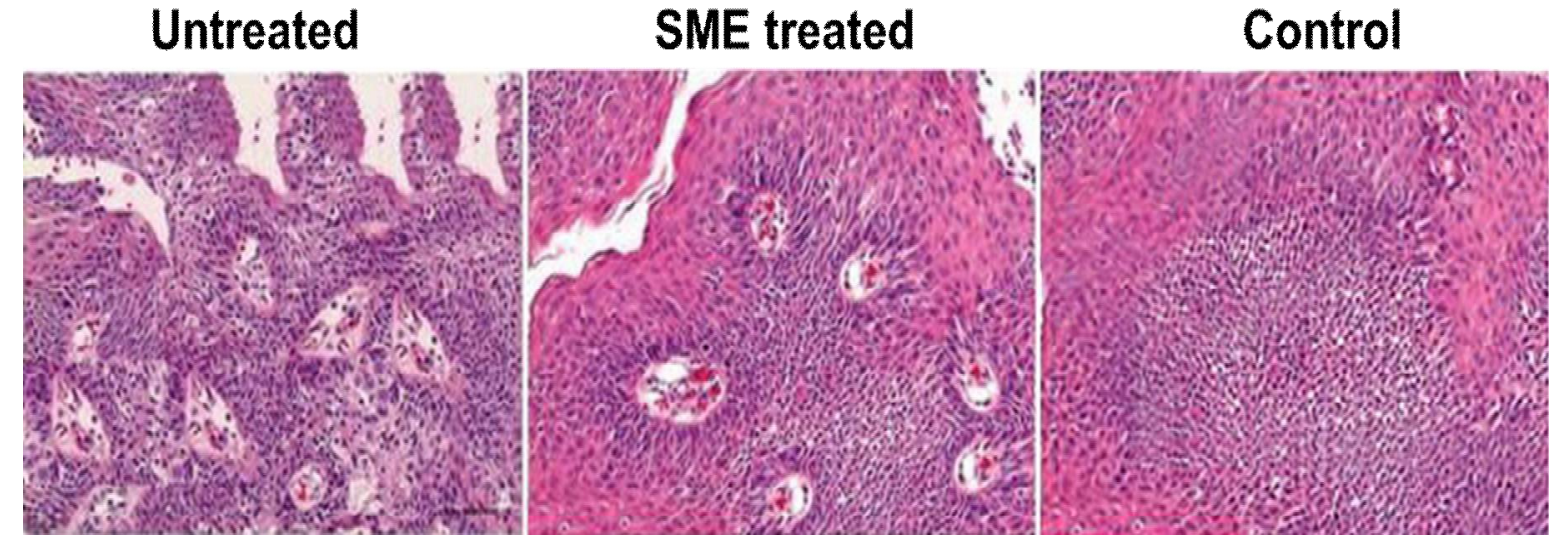

Figure 2: Effect of S. miltiorrhiza root extract on gliocytes in the spinal cord of rats with spinal cord injury. The spinal cord tissues were examined under a microscope following H\&E staining

\section{S. miltiorrhiza root extract increases the expression of PDGF-B}

DAB staining revealed that treatment of the animals with $S$. miltiorrhiza root extract induced an increase in the proportion of PDGF-B-positive cells. Compared to the untreated group, animals in the $S$. miltiorrhiza root extract-treated group showed significantly higher numbers of PDGF-Bpositive cells on day 11 after surgery (Fig. 3). S. miltiorrhiza root extract also resulted in a reduction in the vacuolar region of the spinal cord following injury. Analysis of the expression of mRNA corresponding to PDGF-B revealed a marked increase in the treated group compared to the untreated group (Fig. 4). The expression of mRNA corresponding to PDGF-B in the treated group was 7-fold higher compared to the untreated group.

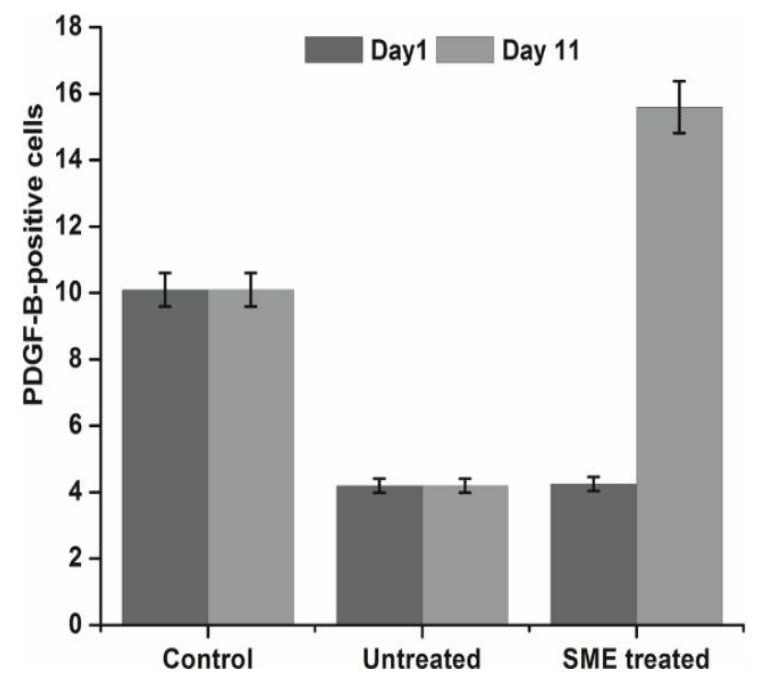

Figure 3: Treatment with $S$. miltiorrhiza root extract increased the proportion of PDGF-B-positive cells in the spinal cord tissues of rats

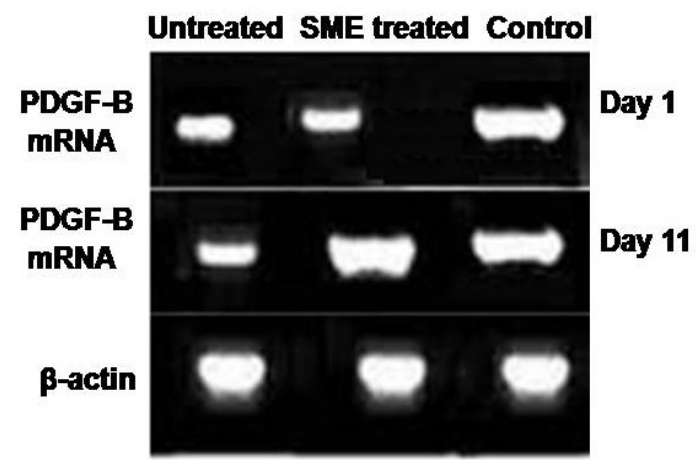

Figure 4: Effect of $S$. miltiorrhiza root extract on the expression of platelet-derived growth factor-B (PDGF$B$ ) in the spinal cord of rats with spinal injury

\section{DISCUSSION}

Extracts of plants such as Lycium barbarum and S. miltiorrhiza have been shown to possess promising neuroprotective activities [12,13]. In the present study, $S$. miltiorrhiza root extract was shown to be beneficial for the treatment of rats with SCI. S. miltiorrhiza root extract induced a significant improvement in recovery compared to untreated rats. Rats in the treatment group showed significantly higher BBB scores and reduced neuronal apoptosis 11 days after surgery. Axons were also found to show regeneration in animals treated with $S$. miltiorrhiza root extract.

The PDGF, which has been demonstrated to bind to NMDA receptors, is secreted by cells in the CNS [10]. It is reported that PDGF plays a vital role in recovery following CNS injury [10]. The mechanism of action of PDGF involves activation of tyrosine kinase receptors, including PDGF- $\alpha R$ and PDGF- $\beta R$. PDGF has also been shown to promote the growth of axons and to maintain the integrity of neurons [11]. PDGF 
isoforms induce mitosis in glial cells, thereby affecting a protective effect on the brain.

It is reported that PDGF protects dopaminergic neurons from toxicity induced by Tat proteins. Increasing PDGF expression may therefore be a promising therapeutic strategy for SCI. The results from the present study revealed that $S$. miltiorrhiza root extract enhances the expression of PDGF-B 10 days after surgery. The improvement in recovery of rats treated with $S$. miltiorrhiza root extract following $\mathrm{SCl}$ may therefore be associated with promotion of PDGF$B$ expression. Reports indicate that the proportion of PDGF-positive cells in the spinal cord of rats is significantly higher following $\mathrm{SCl}$ $[17,21]$. PDGF-B plays an important role in the protection of neurons by inhibiting glutamate transporters in the cell membrane. Furthermore, enhancement of the PDGF-B/receptor signal pathway may rescue neonatal brains at risk of hypoxic-ischemic injury [22].

Thus, we conclude that the root extract of $S$. miltiorrhiza has the capacity to improve recovery from $\mathrm{SCl}$ in rats, via a mechanism involving promotion of PDGF-B expression. Therefore, Salvia miltiorrhiza extract may be of potential therapeutic use for treatment of $\mathrm{SCl}$.

\section{CONCLUSION}

The findings of the present study demonstrate that the root extract of $S$. miltiorrhiza significantly improves recovery, as indicated by increase in locomotor activity, following $\mathrm{SCl}$ injury in a rat model. The results also suggest that $S$. miltiorrhiza root extract has therapeutic potentials for the treatment of neuronal degeneration associated with spinal cord injuries.

\section{DECLARATIONS}

\section{Acknowledgement}

The authors wish to thank the staff of the Department of Orthopaedics and the Spine Division of Xi'an Red Cross Hospital for their support. This study was supported by National Natural Scientific Foundation of China (nos. 10972156 and 10635058) and Specialized Research Fund for the Doctoral Program of Higher Education of China (no. 2010201121049).

\section{Conflict of Interest}

No conflict of interest associated with this work.

\section{Contribution of Authors}

The authors declare that this work was done by the authors named in this article and all liabilities pertaining to claims relating to the content of this article will be borne by them.

\section{REFERENCES}

1. Blight AR. Macrophages and inflammatory damage in spinal cord injury. J Neurotrauma 1992; 9: 83-91.

2. Schmitt C, Miranpuri GS, Dhodda VK, Isaacson J, Vemuganti $R$, Resnick DK. Changes in spinal cord injury-induced gene expression in rat are straindependent. Spine J 2006; 6: 113-119.

3. Hong $Z$, Chen $H$, Hong $H$, Lin L, Wang Z. TSP-1 expression changes in diabetic rats with spinal cord injury. Neurol Res 2009; 31: 878-882.

4. Kose EA, Bakar B, Ayva SK, Kilinc K, Apan A. Neuroprotective effects of racemic ketamine and (S)ketamine on spinal cord injury in rat. Injury 2012; 43: 1124-1130.

5. Bulsara KR, Iskandar BJ, Villavicencio AT, Skene JH. A new millennium for spinal cord regeneration: growthassociated genes. Spine 2002; 27: 1946-1949.

6. Szpara ML, VranizanK, Tai YC, Goodman CS, Speed TP, Ngai J. Analysis of gene expression during neurite outgrowth and regeneration. BMC Neurosci 2007; 8: 100.

7. Busch SA, Silver J. The role of extracellular matrix in CNS regeneration. Curr Opin Neurobiol 2007; 17: 120127.

8. Liu BP, Cafferty WB, Budel SO, Strittmatter SM. Extracellular regulators of axonal growth in the adult central nervous system. Philos Trans $R$ Soc Lond $B$ BiolSci2006; 361: 1593-1610.

9. McGee AW, Strittmatter SM. The Nogo-66 receptor: focusing myelin inhibition of axon regeneration. Trends Neurosci 2003; 26:193 198.

10. Ishii $Y$, Oya $T$, Zheng $L$. Mouse brains deficient in neuronal PDGF receptor-beta develop normally but are vulnerable to injury. J Neurochem 2006; 98: 588-600.

11. Wu QH, Chen WS, Chen QX, Wang JH, Zhang XM. Changes in the expression of platelet-derived growth factor in astrocytes in diabetic rats with spinal cord injury. Chin Med J (Engl) 2010; 123: 1577-1581.

12. Chan HC, Chang RC, Koon Chinglp A. Neuroprotective effects of Lycium barbarum Lynn on protecting retinal ganglion cells in an ocular hypertension model of glaucoma. ExpNeurol2007; 203: 269273.

13. Mi XS, Zhong JX, Chang RC, So KF. Research advances on the usage of traditional Chinese medicine for neuroprotection in glaucoma. J Integr Med 2013; 11: 233240.

14. Cheng TO. Cardiovascular effects of Danshen. Int $J$ Cardiol2007; 121: 922.

15. Qin RA, Yao XX, Huang ZY. Effects of compound danshen tablets on spatial cognition and expression of 
brain beta amyloid precursor protein in a rat model of Alzheimer's disease. J Tradit Chin Med 2012; 32: 6366.

16. Office of Laboratory Animal Welfare/National Institutes of Health. 2002. Public Health Service policy on human care and use of laboratory animals. National Institutes of Health, Bethesda, Maryland. Available from: https://grants.nih.gov/grants/olaw/references/phspol.htm

17. Yang C, Li B, Liu TS, Zhao DM, Hu FA. Effect of electracu $\neg$ puncture on proliferation of astrocytes after spinal cord injury. Zhongguo Zhen Jiu2005; 25: 569572. (In Chinese).

18. Anderson KD, Sharp KG, Steward O. Bilateral cervical contusion spinal cord injury in rats. Experimental neurology 2009; 220: 9-22.

19. Xun C, Hu Y, Lu M, Wang S, Lv D. Study of effect of salvianolic acid $B$ on motor function recovery in rats with spinal cord injury. BioMed research international 2014; doi:10.1155/2014/931850.

20. Kanz L, Luig A, Mielke R, Azemar M, Fauser AA, Schaefer HE. Analysis of c-sis/PDGF-B mRNA expression in megakaryocytic cells of patients with myeloproliferative disorders. Prog Clin Biol Res 1990; 356:287-298.

21. Huang X, Kim JM, Kong TH. GM-CSF inhibits glial scar formation and shows long-term protective effect after spinal cord injury. J Neurol Sci 2009; 277: 87-97.

22. Egawa-Tsuzuki T, Ohno M, Tanaka N. The PDGF Bchain is involved in the ontogenic susceptibility of the developing rat brain to NMDA toxicity. ExpNeurol2004; 186: $89-98$ 